\title{
Value of hysteroscopy prior to uterine artery embolisation for fibroids: a hospital based retrospective study
}

\author{
Geeta B. Krishnamurthy • Ioanis Tsibanakos • \\ Mohsen Moustafa • Mina Karamshi • Neil Davies • \\ Adam Magos
}

Received: 12 April 2012 / Accepted: 16 June 2012 / Published online: 18 July 2012

(C) Springer-Verlag 2012

\begin{abstract}
This study was undertaken to evaluate the value of routine hysteroscopy prior to uterine artery embolisation (UAE) for symptomatic uterine fibroids. The study design used is hospital-based retrospective study, and the setting is in a large teaching hospital. We analysed the hospital record of 115 women who were scheduled to undergo UAE at our institution between January 2008 and April 2011. All women had outpatient hysteroscopic assessment of uterine cavity prior the decision to carry out UAE. The mean uterine size on palpation was 15.4 (standard deviation (SD) 3.5) weeks gestation equivalent. Hysteroscopy was successfully completed in $112(97.4 \%)$. In the women who were hysteroscoped, 50 (44.6\%) had no submucous fibroids; 50 (44.6\%) had type II fibroids, and $12(10.7 \%)$ were found to have type I or 0 fibroids. All 12 women with type 0 or I submucous fibroids were offered hysteroscopic $(n=11)$ or vaginal $(n=1)$ myomectomy prior to UAE, but only four agreed. Of these four cases, two cancelled their planned UAE because of symptomatic improvement. The remaining two women, as well as the eight, who declined surgery, underwent UAE. There were no cases of infection, spontaneous expulsion of a fibroid or the need for surgical intervention in this group. This pilot study shows that hysteroscopy prior to UAE changes management in only a small proportion of cases. Selective hysteroscopy, following MRI scanning, may be a more logical protocol to identify women with intracavitary fibroids, who may benefit from hysteroscopic or vaginal myomectomy.
\end{abstract}

G. B. Krishnamurthy $(\bowtie) \cdot$ I. Tsibanakos $\cdot$ M. Moustafa $•$

M. Karamshi $\cdot$ N. Davies $\cdot$ A. Magos

Department of Gynaecology, Royal Free Hospital,

Pond Street,

London NW3 2QG, UK

e-mail: geeta_om@yahoo.com
Keywords Hysteroscopy · Uterine artery embolisation · Submucous fibroids

\section{Background}

Uterine myomata are the most commonly found gynaecological benign tumours in women of child-bearing age. Uterine fibroids are often asymptomatic, but in $50 \%$ of women, they can cause heavy bleeding, pain, pressure symptoms, subfertility or difficulties with pregnancy and delivery. The standard treatment for large symptomatic uterine fibroids has been hysterectomy, if women have completed their family, or myomectomy, if uterine conservation is required.

During the mid-1990s, uterine artery embolisation (UAE) was introduced as a minimally invasive uterus-conserving alternative to hysterectomy and myomectomy. Embolising the uterine blood supply had been used earlier to manage post-partum haemorrhage and as treatment of uterine arterio-venous malformations, but it was the desire to reduce intraoperative haemorrhage at open myomectomy by Professor Jacques Ravina, which serendipitously led to the use of UAE as primary management of this common condition once it was realised that the embolisation lets to fibroid shrinkage and symptomatic improvement $[1,2]$. Many studies since have confirmed both the benefits and risks of UAE $[3,4]$.

One of the most feared complications of UAE is postembolisation uterine sepsis, a complication which may necessitate hysterectomy or, worse, can have a fatal outcome [5]. The risk of post-UAE sepsis has been estimated at 1$2 \%$, but is greater in presence of submucous fibroids. Spontaneous and painful expulsion of submucous fibroids is another possible complication which affects up to $10 \%$ of patients [6]. Some women experience offensive vaginal 
discharge and the passage of necrotic tissue for several weeks after UAE [7-9], whilst fibroid expulsion is often spontaneous, some need surgical evacuation of detached submucous fibroids.

Several studies have shown saline infusion sonography as effective, cheaper and less invasive method in diagnosing submucous fibroids [10-12]. Magnetic resonance imaging (MRI) is a standard practice in many units to evaluate the uterus prior to UAE, partly to exclude adenomyosis and pedunculated myomas, but also to evaluate distortion of the uterine cavity by submucous fibroids $[13,14]$. The evidence for routine hysteroscopy prior to UAE, to exclude submucous fibroids, is limited.

We have been carrying out routine outpatient hysteroscopy in all women to diagnose and treat submucous fibroids prior to UAE. In this retrospective study, we wanted to assess the value of such a strategy in this patient group and assess if the hysteroscopy had changed the plan of management in those women who were otherwise scheduled to have uterine artery embolisation, for example, this could be scheduling women for hysteroscopic resection of submucous fibroids prior to UAE. Though many units consider MRI as one of the preliminary investigations, very few units carry out routine hysteroscopic assessment of uterine cavity.

\section{Patients and methods}

We analysed the medical records of 115 women who underwent UAE for uterine fibroids at the Royal Free Hospital between January 2008, when we introduced universal screening of women awaiting UAE by hysteroscopy, and April 2011. All women, who had multiple medium to large uterine fibroids, preferred UAE to surgical management; $80 \%$ of them suffered from menorrhagia, and $20 \%$ had mixed symptoms like menorrhagia, pelvic pain and pressure symptoms.

Patient selection for UAE was carried out by multidisciplinary team, including a gynaecologist and an interventional radiologist. We selected women who had completed their family and or at increased risk from surgery or where UAE was their first choice than surgery. MRI and routine outpatient hysteroscopy were the standard investigations in our unit prior to UAE.
We obtained a written consent prior to all outpatient hysteroscopy procedures. When we compiled the data to study the value of hysteroscopy, we did not obtain hospital ethical committee approval due to (1) the retrospective nature of the study, and (2) the study is looked upon as Service Development.

Hysteroscopy was done in the Outpatient Hysteroscopy Clinic. We used a standard $2.9 \mathrm{~mm} 30^{\circ}$ rigid hysteroscope (Karl Storz, Tuttlingen, Germany) with N/saline for uterine distension at a starting pressure of $150 \mathrm{mmHg}$. A "no touch" (vaginoscopic) technique was used whenever possible [15]. Endometrial sampling with an $\mathrm{H}$ Pipelle was taken in selected cases of women with irregular or prolonged bleeding who were $>45$ years old [16].

Where indicated, minor outpatient procedures, such as polypectomy, were carried out with mechanical instruments or a mini-resectoscope (Roberts Surgical Healthcare, Kidderminster, England) [17]. Those found to have type 0 or 1 submucous fibroids were offered hysteroscopic or vaginal myomectomy prior to UAE.

\section{Findings}

A total of 115 women with symptomatic fibroid uterus were scheduled for UAE during the study period. The average age of the group was 44.6 (SD 4.09) years. The mean uterine size on palpation was 15.4 (SD 3.5) weeks gestation equivalent, though the uterine size ranged between 12 and 32 weeks.

Of the patients, 112 (97.4\%) had successful hysteroscopic assessment of the uterine cavity, the investigation having to be abandoned in $3(2.5 \%)$ due to pain and vasovagal attack. The hysteroscopic findings of the women, who underwent hysteroscopy, are summarised in Table 1. Just over half the patients (55.4\%) had submucous fibroids, but only $12(10.7 \%)$ were mostly intracavitary (type 0 or I). In addition, six of the patients were diagnosed with endometrial polyps; all polyps were removed at the same time. One woman required removal of a Mirena intrauterine system prior to UAE to minimise the risk of post-embolisation sepsis.

MRI results of 12 women with hysteroscopically detected submucous fibroids were reviewed. In $75 \%$ of women $(n=9)$,

Table 1 Hysteroscopic findings

\begin{tabular}{lllll}
\hline Type of fibroid on hysteroscopy & Number of women & $\begin{array}{l}\text { Surgical resection } \\
\text { of fibroid }\end{array}$ & Infection & $\begin{array}{l}\text { Expulsion } \\
\text { of fibroid }\end{array}$ \\
\hline $\begin{array}{l}\text { No submucous fibroids } \\
\text { (type II and deeper fibroids) }\end{array}$ & 50 & 0 & 1 & 0 \\
Type 0 or I & 12 & 4 & 0 & 0 \\
Type II & 50 & 0 & 1 & 0 \\
\hline
\end{tabular}


the MRI was suggestive of submucous fibroids. In $25 \%$ of women $(n=3)$, MRI failed to pick up resectable submucous fibroids.

Of the 12 women with type 0 or I fibroids, 4 agreed to undergo hysteroscopic myomectomy and 1 vaginal myomectomy prior to UAE. The hysteroscopic myomectomies were completed successfully, but the vaginal myomectomy was abandoned as the fibroid was too high in the uterine cavity for vaginal excision. Two of the four women, who underwent hysteroscopic myomectomy, subsequently cancelled their planned UAE as their symptoms improved with hysteroscopic resection of fibroids alone. The remaining ten patients underwent UAE. There were no cases of infection, spontaneous expulsion of a fibroid or the need for surgical intervention in this subgroup; however, uterine sepsis was diagnosed in two women with type II, and deeper fibroids, in those who underwent UAE (Table 1).

All 103 women with normal uterine cavities or type II submucous fibroid at hysteroscopy also underwent UAE. Two of this subgroup was admitted to hospital with infection, approximately 2 weeks after the embolisation. They were managed conservatively with antibiotics, and none required hysterectomy.

\section{Conclusion}

Our protocol of carrying out routine diagnostic hysteroscopy prior to UAE, to identify patients with type 0 or I fibroids, does not seem to be worthwhile. Although we identified $12(10.7 \%)$ women with submucous fibroids which were wholly or mostly in the uterine cavity, only 5 agreed to undergo hysteroscopic or vaginal myomectomy, and only 2 ultimately cancelled their embolisation because of symptomatic improvement. None of the remaining ten had any problems after embolisation in terms of infection or fibroid expulsion. As a result, we no longer offer routine diagnostic hysteroscopy to patients requesting UAE just to confirm or exclude the presence of intracavitary fibroids.

MRI in our study was sensitive in picking up type 0-1 submucous fibroids in $75 \%$ of women. In $25 \%$ of women, type 0-1 submucous fibroids were missed, but were reported as type II fibroids. Also, MRI was not specific for women with endometrial polyps.

Apart from the 12 patients with submucous fibroids, we also found that 6 women had endometrial polyps on routine hysteroscopy. The polyps were asymptomatic and proved to be benign on histology. There is debate regarding the need to remove benign asymptomatic polyps, and for this reason, we doubt if routine hysteroscopy can be justified just for the purposes of excluding such intrauterine lesions [18, 19].

Based on our results, a more logical protocol for assessing patients prior to UAE would be selective hysteroscopy in those women with a suspicion of intracavitary fibroids on MRI scanning. MRI scanning is routine prior to UAE in our hospital, and earlier studies have shown that MRI, hysterosonographic examination and diagnostic hysteroscopy were equally effective for evaluation of uterine cavity. Even if not all such patients elect to undergo hysteroscopic (or vaginal) excision of the relevant fibroid, our experience shows that some will, and this can be associated with symptomatic relief in some, such that UAE becomes unnecessary. This study has led to change in our clinical practice, as we do now selective hysteroscopy prior to UAE since June 2011.

Acknowledgments We would like to thank all the staff at hysteroscopy clinic and intervention radiology for their help in providing the data for analysis. This study was presented as oral presentation at 20th annual congress of European Society for Gynaecological Endoscopy (ESGE) at London in September 2011.

Conflict of interest None.

\section{References}

1. Ravina JH, Herbreteau D, Ciraru-Vigneron N, Bouret JM, Houdart $\mathrm{H}$, Aymard A et al (1995) Arterial embolisation to treat uterine myomata. Lancet 346:671-672

2. Ravina J, Bouret J (1997) Cirary-Vigneron N application of particulate arterial embolization in the treatment of uterine fibromyomata. Bull Acad Natl Med 181:233-243

3. Hirst A, Dutton S, Wu O, Briggs A, Edwards C, Waldenmaier L et al (2008) The HOPEFUL Study: a multi-centre retrospective cohort study comparing the efficacy, safety and cost-effectiveness of hysterectomy and uterine artery embolisation (UAE) for the treatment of symptomatic uterine fibroids. NHS Health Technology Assessment Programme. HTA Ref: 03/60/01

4. Rajan DK, Beecroft JR, Clark TW, Asch MR, Simons ME, Kachura JR, Sved M (2004) Sniderman KW Risk of intrauterine infectious complications after uterine artery embolization. J Vasc Interv Radiol 15(12):1415-1421

5. Martino MA, Garcia JE, Deutsch A, Borges E, Hoffman MS (2005) Sepsis leading to emergent hysterectomy after uterine artery embolization. J Gynecol Surg 21(4):173-175. doi:10.1089/ gyn.2005.21.173

6. Radeleff B, Eiers M, Bellemann N, Ramsauer S, Rimbach S, Kauczor HU, Richter GM (2010) Expulsion of dominant submucosal fibroids after uterine artery embolisation. Eur J Radiol 75(1): e57-e63, Epub 2009 Aug 18

7. Felemban A, Stein L, Tulandi T (2001) Uterine restoration after repeated expulsion of myomas after uterine artery embolization. J Am Assoc Gynecol Laparosc 8(3):442-444

8. Park HR, Kim MD, Kim NK, Kim HJ, Yoon SW, Park WK, Lee MH (2005) Uterine restoration after repeated sloughing of fibroids or vaginal expulsion following uterine artery embolization. Eur Radiol 15(9):1850-1854, Epub 2005 Feb 24

9. Redecha M Jr, Holomán K, Javorka V, Mizícková M, Ferianec V, Papcun P, Krizko M Jr, Redecha M Sr (2009) Myoma expulsion after uterine artery embolisation. Arch Gynecol Obstet 280 (6):1023-1024, Epub 2009 Mar 25

10. Cepni I, Ocal P, Erkan S, Saricali FS, Akbas H, Demirkiran F, Idil M, Bese T (2005) Comparison of transvaginal sonography, saline infusion sonography and hysteroscopy in the evaluation of uterine cavity pathologies. Aust N Z J Obstet Gynaecol 45(1):30-35 
11. Salim R, Lee C, Davies A, Jolaoso B, Ofuasia E, Jurkovic D (2005) A comparative study of three-dimensional saline infusion sonohysterography and diagnostic hysteroscopy for the classification of submucous fibroids. Hum Reprod 20(1):253-257, Epub 2004 Oct 21

12. Bartkowiak R, Kaminski P, Wielgos M, Bobrowska K (2006) The evaluation of uterine cavity with saline infusion sonohysterography and hysteroscopy in infertile patients. Neuro Endocrinol Lett 27(4):523-528

13. Dueholm M, Lundorf E, Hansen ES (2001) Evaluation of the uterine cavity with MRI, transvaginal sonography, hysterosonographic examination and diagnostic hysteroscopy. Fertil Steril 76 (2):350-357

14. Dueholm M, Lundorf E (2002) Sorensen JS Reproducibility of evaluation of the uterus by transvaginal sonography, hysterosonographic examination, hysteroscopy and MRI. Hum Reprod 17 (1):195-200
15. Agostini A, Crochet P, Blanc K, Collette E, Cravello L, Blanc B (2006) Vaginoscopic hysteroscopy. Gynecol Obstet Fertil 34 (5):420-422

16. Di Spiezio Sardo A, Sharma M, Taylor A, Buck L, Magos A (2004) A new device for "no touch" biopsy at "no touch" hysteroscopy: the H Pipelle. Am J Obstet Gynecol 191:157-158

17. Papalampros P, Gambadauro P, Papadopoulos N, Polyzos D, Chapman L, Magos A (2009) The mini-resectoscope: a new instrument for office hysteroscopic surgery. Acta Obstet Gynecol Scand 88(2):227-230

18. Lieng M, Istre O, Qvigstad E (2010) Treatment of endometrial polyps: a systematic review. Acta Obstet Gynecol Scand 89 (8):992-1002

19. Nogueira AA, Dos Reis FJC, Silva JCRE, Netto OBP, de Freitas Barbosa H (2007) Endometrial polyps: a review. J Gynecol Surg 23(3):111-116 